\title{
Basal and starvation-induced autophagy mediates parasite survival during intraerythrocytic stages of Plasmodium falciparum
}

\author{
Shiny Joy ${ }^{1}$, Lavanya Thirunavukkarasu', Palak Agrawal', Archana Singh², B. K. Chandrasekhar Sagar ${ }^{3}$, \\ Ravi Manjithaya (1) ${ }^{1}$ and Namita Surolia ${ }^{1}$
}

\begin{abstract}
The precise role of autophagy in $P$. falciparum remains largely unknown. Although a limited number of autophagy genes have been identified in this apicomplexan, only PfAtg8 has been characterized to a certain extent. On the basis of the expression levels of PfAtg8 and the putative PfAtg5, we report that the basal autophagy in this parasite is quite robust and mediates not only the intraerythrocytic development but also fresh invasion of red blood cells (RBCs) in the subsequent cycles. We demonstrate that the basal autophagy responds to both inducers and inhibitors of autophagy. In addition, the parasite survival upon starvation is temporally governed by the autophagy status. Brief periods of starvation, which induces autophagy, help survival while prolonged starvation decreases autophagy leading to stalled parasite growth and reduced invasion. Thus, starvation-induced autophagy is context dependent. Importantly, we report characterization of another autophagy marker in this parasite, the putative PfAtg5 (Pf3D7_1430400). PfAtg5 is expressed in all the intraerythrocytic stages and partially colocalizes with ER, mitochondria, apicoplast and PfAtg8. It is also present on the double membrane bound vesicles. Altogether, these studies pave way for the detailed dissection of $P$. falciparum autophagy machinery and insights into molecular and functional characterization of its players for developing new therapeutics as antimalarials.
\end{abstract}

\section{Introduction}

Macroautophagy (hereafter autophagy) in eukaryotes is a conserved catabolic process, whereby cytosolic constituents such as proteins and organelles are captured in double membrane vesicles called autophagosomes and are transported to lysosomes for degradation. The amino acids and other macromolecular constituents thus generated in lysosomes are recycled to maintain cellular

\footnotetext{
Correspondence: Ravi Manjithaya (ravim@jncasr.ac.in) or

Namita Surolia (surolia@jncasr.ac.in)

${ }^{1}$ Molecular Biology and Genetics Unit, Jawaharlal Nehru Centre for Advanced Scientific Research, Jakkur, Bangalore 560064, India

${ }^{2}$ CSIR-Institue of Genomics and Integrative Biology, Room 223, Sukhdev Vihar, Mathura Road, New Delhi 110025, India

Full list of author information is available at the end of the article.

Edited by A. Rufini
}

homeostasis ${ }^{1}$. Autophagy thus serves as a response mechanism for survival under stress conditions such as starvation, hypoxia, high temperatures, differentiation, protein metabolism, etc ${ }^{2}$.

The complex life cycle of human malaria parasite $P$. falciparum consists of three major stages; in mosquito gut, human liver and blood. During its $48 \mathrm{~h}$ asexual intraerythrocytic cycle, the parasite development progresses through ring, trophozoite and schizont stages producing upto 32 daughter cells (merozoites) that can reinvade fresh red blood cells. Some of the released merozoites develop into male and female gametocytes, which when taken up by a female mosquito during a blood meal undergo sexual reproduction in the mosquito midgut. The sporozoites which develop in the mosquito

\section{(c) 2018 The Author(s).}

(c) (i) Open Access This article is licensed under a Creative Commons Attribution 4.0 International License, which permits use, sharing, adaptation, distribution and reproduction in any medium or format, as long as you give appropriate credit to the original author(s) and the source, provide a link to the Creative Commons license, and indicate if changes were made. The images or other third party material in this article are included in the article's Creative Commons license, unless indicated otherwise in a credit line to the material. If material is not included in the article's Creative Commons license and your intended use is not permitted by statutory regulation or exceeds the permitted use, you will need to obtain permission directly from the copyright holder. To view a copy of this license, visit http://creativecommons.org/licenses/by/4.0/. 
are released during the next mosquito bite and reach human hepatocytes. The sporozoites undergo another asexual division in liver and release merozoites into the blood stream, thus completing the cycle. Thus, throughout the life cycle, the parasite encounters phases of nutritional limitations and other stresses. Understanding pathways such as autophagy in this important parasite therefore may give insights towards developing novel antimalarials.

Using bioinformatics analyses, a limited number of autophagy proteins in P. falciparum genome have been identified $^{3,4}$, but the precise functions of these putative proteins and the role of autophagy in this organism till date remains unanswered. Atg8, the autophagosome marker is the major autophagy protein that has been studied both in P. berghei as well as in P. falciparum in certain detail. Studies that investigated localization of this protein in $P$. falciparum have not yielded a clear picture. PfAtg8 has been shown to localize exclusively or partially to the apicoplast ${ }^{5,6}$, in the host $\mathrm{RBCs}^{6}$ and also to be present as puncta in the parasite cytosol ${ }^{4,6-8}$. The localization of PfAtg8 is shown to be unperturbed upon pharmacological disruption of PtdIns3K by wortmannin ${ }^{5}$, $\mathrm{CQ}^{4,6}$, inhibition of putative TOR by rapamycin $^{6}$ or manipulation of the food vacuole $\mathrm{pH}^{4}$. Further, the absence of autophagy induction upon starvation is attributed to the lack of the negative regulator, TOR in Plasmodium $^{4,6}$, although, Langsley et al. report ${ }^{8}$ increase in colocalization of PfAtg8 and PfRab7 upon starvation.

Studies in $P$. berghei have indicated that in the liver stages, autophagy-like mechanism may be prevalent during metamorphosis of the sporozoites into merozoites to remove unnecessary organelles ${ }^{9}$. While in P. falciparum, it has been proposed that autophagy has evolved to perform multiple functions such as apicoplast biogenesis, protein turnover, cellular differentiation and partitioning into daughter cells ${ }^{6}$, but no experimental evidence exists for this hypothesis. The role of autophagy pathway during intraerythrocytic cycle thus has not been unequivocally established in P. falciparum. Furthermore, the localization and expression of PfAtg8 is controversial and other autophagosome markers such as PfAtg 5 have not been explored yet. For these reasons, we initiated our studies to decipher the functional role of autophagy in P. falciparum during its erythrocytic stages and to characterize the putative PfAtg5 as a player of Plasmodium autophagy pathway. We show that pharmacological inhibition of basal autophagy leads to compromised development and reinvasion abilities of the parasite. In addition, we also demonstrate that starvation-induced autophagy is temporally governed. Moderate starvation enables the parasite survival but prolonged starvation results in cell death. At the molecular level, both PfAtg8 and PfAtg5 appear as punctae resembling autophagosomes and their expression increase transiently upon brief starvation but later decrease with time which correlates with parasite survival and death respectively. Our study thus identifies a context dependent role of starvation-induced autophagy in this parasite.

\section{Results}

\section{Inhibition of basal autophagy results in reduced reinvasion} and PfAtg8 expression

A basal level of autophagy is responsible for maintaining cellular homeostasis in almost all the living organisms ${ }^{1}$. Despite a number of studies carried out recently, the role of autophagy in $P$. falciparum is poorly understood. To investigate the precise role of autophagy in P. falciparum, we tested the ability of blood stage parasites to invade fresh RBCs as an indicator of growth and development in presence or absence of the known autophagy inhibitor, 3methyl adenine (3-MA). Synchronized schizont stage parasites when incubated with 3 -MA showed $40 \%$ less rings compared to control in the ensuing invasion (Fig. 1a, b). Further, the expression levels of the autophagosome marker, PfAtg8 in 3-MA treated parasites (trophozoite stage) were reduced by 3 -fold (Fig. 1c). Immunocytochemical analysis using PfAtg8 antibodies revealed the presence of Atg8 labeled vesicles (puncta)only in the parasite cytosol. The number of these Atg8 vesicles were reduced $50 \%$ in the 3-MA treated parasites as compared to control (Fig. 1d) corroborating our immunoblot analysis. To visualize these puncta more clearly, we used super resolution microscopy (Three-Dimensional Structured Illumination Microscopy, 3D-SIM). Numerous small vesicles labeled with PfAtg8, resembling autophagosomes, were seen in the parasite cytosol in all the three stages, that is, ring, trophozoite, and schizont (Supplementary Figure S1). In addition, electron micrographs of starved parasites revealed the presence of PfAtg8 on double/multiple membrane vesicles reminiscent of autophagosomes near and within the food vacuole (Supplementary Figure S2). As these vesicles have been shown to associate with the apicoplast, we studied the colocalization of PfAtg8 vesicles with the apicoplast marker, Single-Stranded DNA Binding protein (SSB). These markers showed marginal colocalization (Supplementary Video S1 A and B). As 3-MA inhibits PI3Kinase which is upstream of the autophagy pathway and is also implicated in other functions, we studied the effect of MRT 68921, the only known specific autophagy inhibitor ULK $1^{10}$. Activation of ULK1 initiates autophagy. PfAtg1 gene ID Pf3D7_1450000 has been predicted to be a Plasmodium homolog of human ULK $1^{11}$. We modeled the structure of PfAtg1 with bound MRT 68921 (Supplementary Figure $3 \mathrm{~A}, \mathrm{~B})$. The docked inhibitor fits well into the binding pocket with an interaction energy of $-7.8 \mathrm{kcal} / \mathrm{mol}$. Superposition of the PfAtg1 model along with the docked 
A

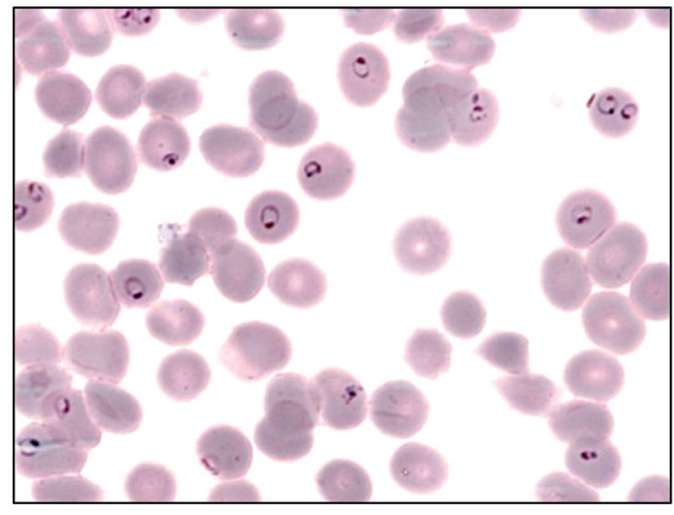

B

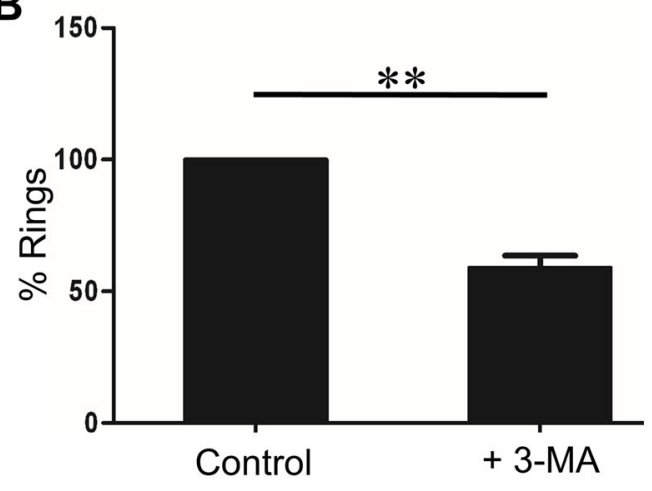

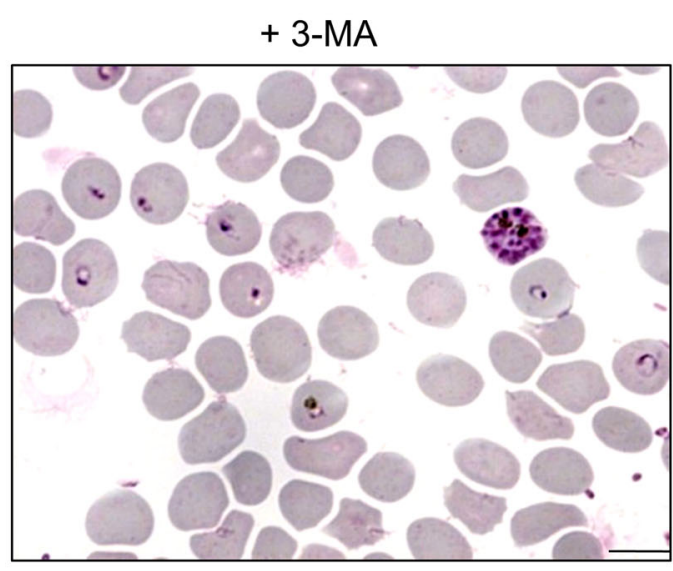

C

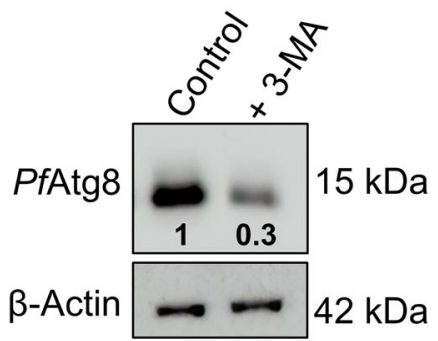

D

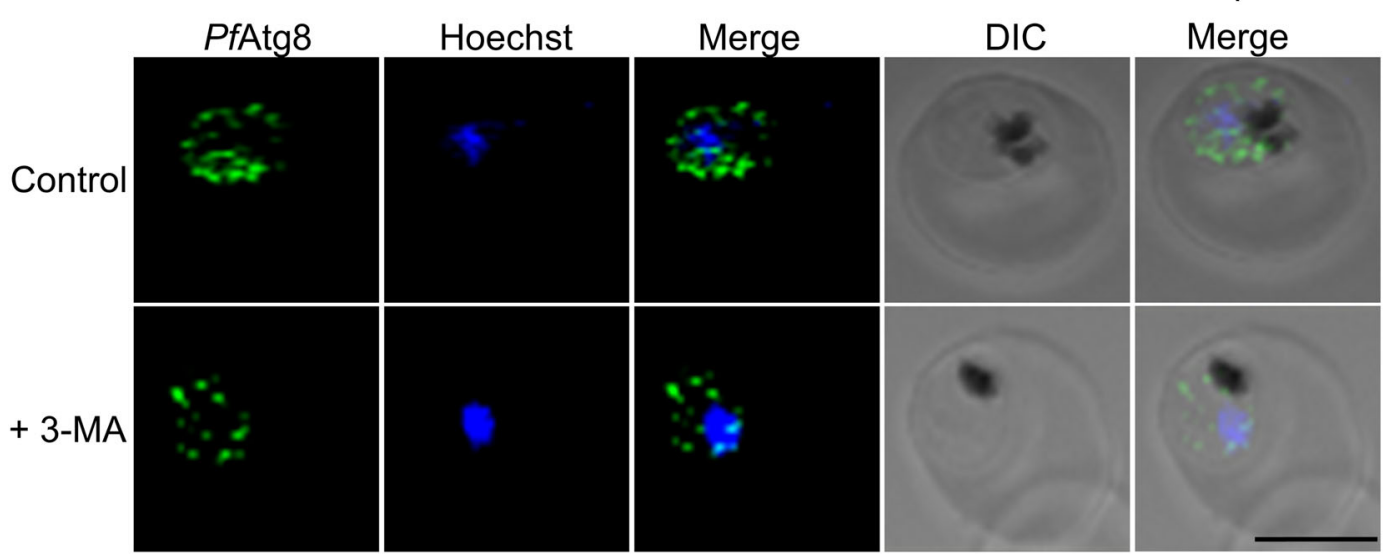

Fig. 1 Inhibition of basal autophagy results in reduced reinvasion and PfAtg8 expression. a Giemsa stained smears showing rings in control and 3-MA treated parasites. Highly synchronized parasites at late schizonts stage (38 h.p.i) were incubated in complete medium (control) with or without 3-MA ( $5 \mathrm{mM})$ for $2 \mathrm{~h}$, and invasion was monitored by counting number of rings in the next cycle. Scale bar: $10 \mu \mathrm{m}$. $\mathbf{b}$ Graph representing percent rings in control and 3-MA treated parasites. The data presented are mean of 5 individual experiments. Error bars show the standard deviation. ${ }^{*} P<0.05$. c Immunoblot analysis of PfAtg8 expression levels. Lysates were prepared from tightly synchronized parasites at trophozoite stage incubated in complete medium with or without 3-MA for $2 \mathrm{~h}$. Immunoblot was probed using custom generated anti-PfAtg8 antibodies. $\beta$-actin was probed as loading control. Number below the bands indicate fold difference as compared to the normalized control. Uncropped blots are shown in Supplementary Figure 5. d PfAtg8 immunofluorescence staining in control and 3-MA treated parasites. Hoechst was used as a DNA marker. Scale bar: $5 \mu \mathrm{m}$ 
MRT68921 on the crystal structure of Human ULK1 kinase in complex with an inhibitor (PDB code: $4 \mathrm{WNO}$ ) shows that MRT68921 fits well into the binding site (Supplementary Figure 3A). The binding site residues Lys70, Asp190, Phe191 are conserved between the two proteins and the gate keeper residue methionine is replaced by Leu123 in PfAtg1. In the ULK1 kinase, a few hydrogen bonds are formed between the protein and the ligand. However, similar hydrogen bonds cannot occur with MRT68921 as the chemical structures of the two inhibitors are different. MRT68921 is likely to make different set of hydrogen bonds or water bridges with PfAtg1, and the additional long chain will enhance binding by making more van der Waals interactions with the protein. We also examined the effect of this inhibitor on the parasite invasion. Our results show that MRT86921 inhibitor (200 nM) completely inhibits the invasion of the fresh erythrocytes (Supplementary Figure 3B). Altogether, these results verify our proposition that basal autophagy mediates the invasion in P. falciparum.

\section{Starvation-induced autophagy mediates parasite invasion}

Nutrient deprivation invariably induces autophagy ${ }^{12}$. However, absence of autophagy induction by starvation has been reported in P. falciparum by several groups ${ }^{4,6}$. Since in our studies 3-MA-treated parasites showed reduced invasion and decreased PfAtg8 expression, indicating that parasite autophagy can be modulated, we next addressed whether the parasite responds to nutrient limitations and if autophagy can be induced by starvation. Interestingly, late schizonts ( 38 h.p.i) when incubated in starvation medium for $2 \mathrm{~h}$ did not lead to a decrease in invasion competence as envisaged by the number of rings in the ensuing cycle (Fig. 2a, b). However, when the parasites were grown in starvation media along with 3MA, 40\% less rings were observed. The modulation of autophagy by starvation or 3-MA was also corroborated by monitoring PfAtg8 expression by immunoblotting and immunofluorescence PfAtg8 expression increased 2-fold upon starvation, while this rise was reduced to near basal levels in presence of 3-MA (Fig. 2c, d). These results demonstrate that autophagy in P. falciparum is induced in response to nutrient limitations mediating reinvasion of host RBCs.

\section{Starvation-induced autophagy is context dependent and determines parasite survival}

As short-term starvation-induced autophagy sustained parasite development and invasion, we further probed if prolonged starvation too could support the erythrocytic cycle. We first determined the autophagy status by monitoring the PfAtg8 expression levels at the trophozoite stage. As seen for $2 \mathrm{~h}$ of starvation, PfAtg8 expression remained high till $4 \mathrm{~h}$ (Fig. 3a). The parasites also appeared morphologically healthy till $4 \mathrm{~h}$ of starvation (Fig. 3b). Interestingly, parasites when starved for longer periods $(6 \mathrm{~h})$, showed decreased PfAtg8 expression as well as retarded growth [Fig. 3a, c $6 \mathrm{~h}$, Control and Starvation (Starv.), and Supplementary Figure S3], indicating that starvation-induced autophagy is temporally governed and is context dependent. Thus, while brief starvation $(2-4 \mathrm{~h})$ induces autophagy and maintains parasite development, prolonged starvation ( $6 \mathrm{~h}$ and beyond) leads to retarded growth. These results led us to further assess the effect of long-term starvation and autophagy decrease (perhaps due to exhaustion of the autophagic process) on parasite development and invasion. The effect of starvation was apparent at $6 \mathrm{~h}$ itself. When starvation or autophagy inhibition was extended upto $6 \mathrm{~h}$, reduced parasitemia, appearance of abnormal parasite morphology and decrease in PfAtg8 expression was apparent. While morphology of control parasites appeared as evenly stained, well rounded, large, full trophozoites with clear and concentrated hemozoin as well as healthy schizonts (Control, $6 \mathrm{~h}$ in Fig. 3c, Supplementary Figure S3 and Control, $6 \mathrm{~h}$ in Fig. 3d), $6 \mathrm{~h}$ starved parasites were smaller, with uneven staining (Starvation (Starv.), $6 \mathrm{~h}$ in Fig. 3c and Supplementary Figure S3). At this time point, the parasitemia remained same even when basal or starvationinduced autophagy was decreased (Fig. 3d). However, the proportion of healthy parasites decreased considerably in the starved and 3-MA treated population. These treated parasites appeared as shrunken late trophozoites with uneven staining (Starv., Control + 3-MA, Starv. + 3-MA, 6 h, Fig. 3c, d and Supplementary Figure S3). Beyond 6 h, starvation and 3-MA treatment progressively increased the population of unhealthy parasites. After $12 \mathrm{~h}$ starvation, while control parasites developed into late schizonts and rings, $80 \%$ of the total starved parasites appeared as shrunken rings or trophozoites. The parasitemia was 58\% less in starved parasites compared to the control (Control, Starv., 12 h, Fig. 3c, Supplementary Figure S3 and 3e). Parasites incubated in starvation medium + 3-MA for 12 $\mathrm{h}$ remained as vacuolated trophozoites and there was 73\% drop in total parasitemia compared to control, while parasites incubated in complete medium + 3-MA showed $69 \%$ decrease in total parasitemia (Control + 3-MA, Starv. + 3-MA, 12 h, Fig. 3c, Supplementary Figure S3 and $3 e)$. After $24 \mathrm{~h}$ starvation, all the parasites grown in control medium progressed to ring stage while the parasites grown in starvation medium showed abnormal morphology with uneven staining. Parasites incubated in starvation medium + 3-MA, were darkly stained and condensed, while parasites incubated in complete medium + 3-MA appeared unevenly stained with jagged borders (Control, Starv., Starv. + 3-MA, Control + 3-MA, $24 \mathrm{~h}$, Fig. 3c, Supplementary Figure S3). As expected, the parasitemia in untreated parasites increased to $18 \%$. In 


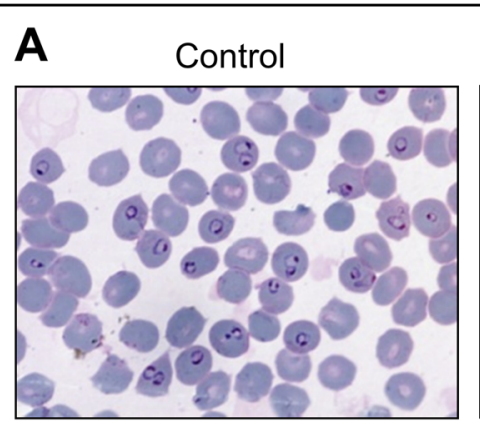

B

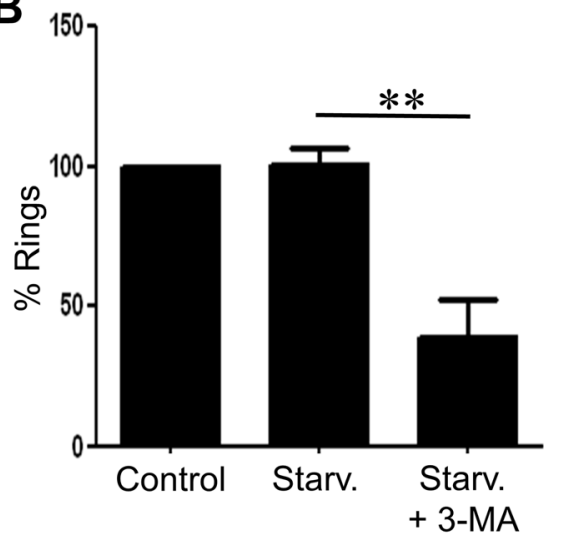

Starvation

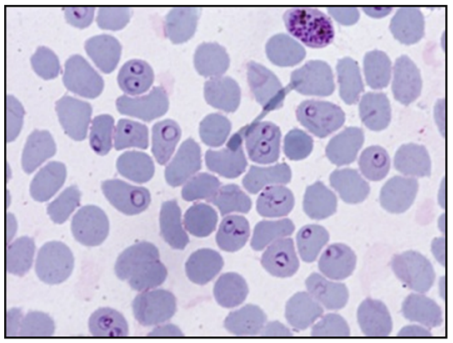

Starvation + 3-MA

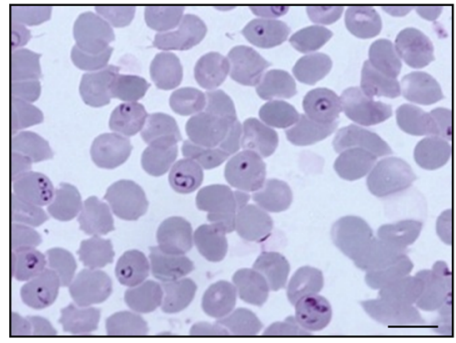

C

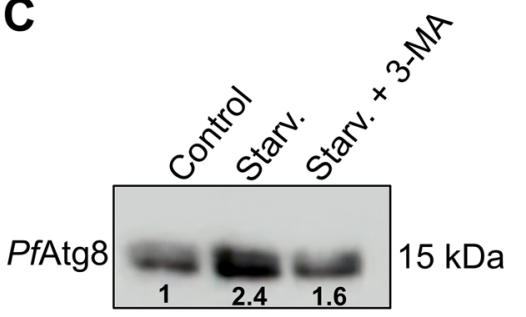

B-Actin

D

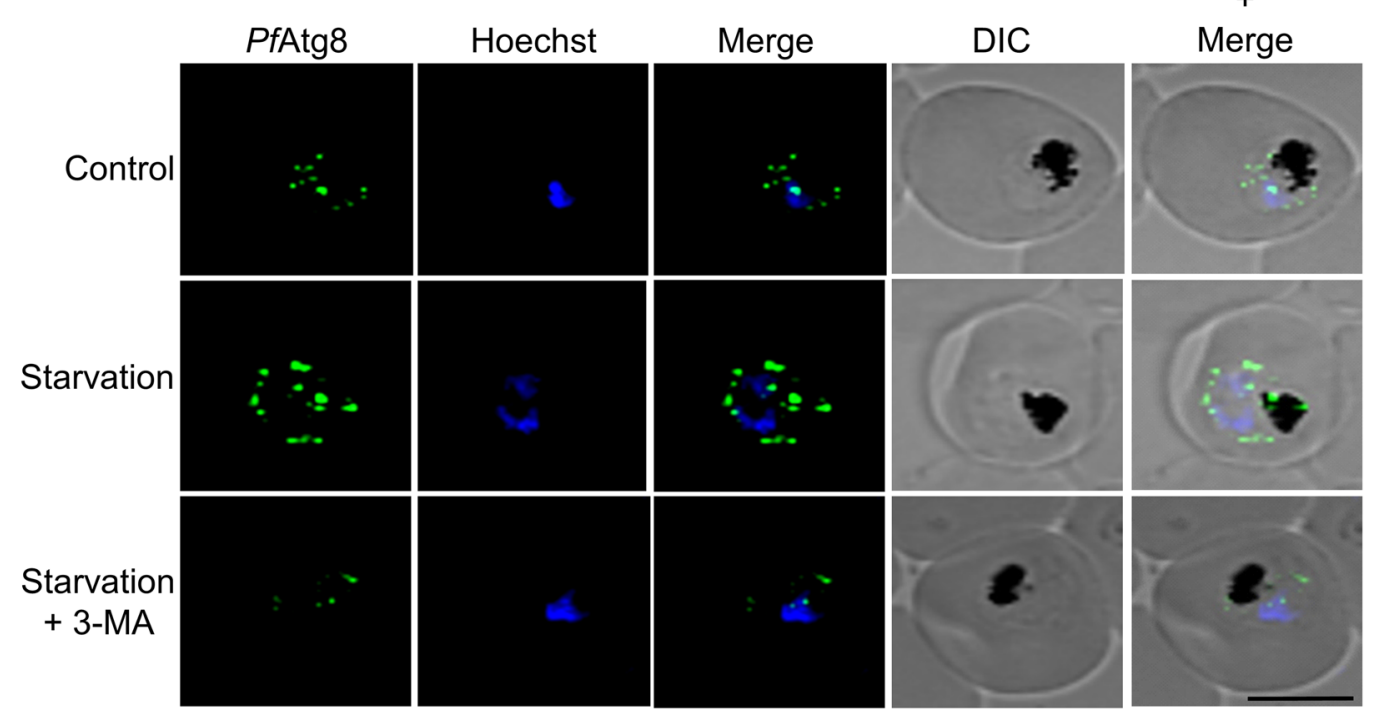

Fig. 2 Starvation-induced autophagy mediates parasite invasion. a Giemsa stained smears showing rings in ensuing invasion of fresh RBCs in control, starved and starved parasites treated with 3-MA (5 mM, 2 h). Highly synchronized parasites at late schizont stage (38 h.p.i) were incubated in control, starvation medium (complete medium devoid of serum and amino acids) with or without 3-MA (5 mM) for $2 \mathrm{~h}$, and number of rings in parasites were counted in ensuing invasion. Scale bar: $10 \mu \mathrm{m}$. b Graph representing percent rings in parasites grown in control, starvation or starvation medium with 3-MA. The data represented are mean of 5 individual experiments (200 parasites counted in each experiment). Error bars show standard deviation. ${ }^{* *} P<0.05$. c Immunoblot analysis of PfAtg8 expression levels. Lysates from trophozoites were prepared from control or starved parasites treated with or without 3-MA and probed using anti-PfAtg8 antibodies. $\beta$-actin was used as loading control. Number below the bands indicate fold difference compared to the normalized control. Uncropped blots are shown in Supplementary Figure 5. $\mathbf{d}$ Immunofluorescence staining of PfAtg8 in parasites incubated for $2 \mathrm{~h}$ in control, starvation or starvation medium with 3-MA (5 mM). Hoechst was used as a DNA marker. Scale bar: $5 \mu \mathrm{m}$ 
A

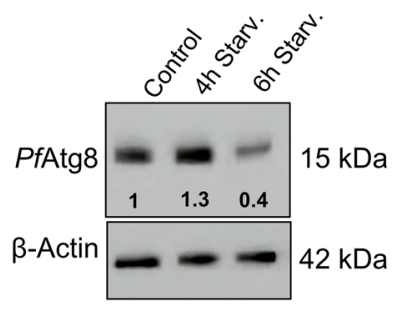

B
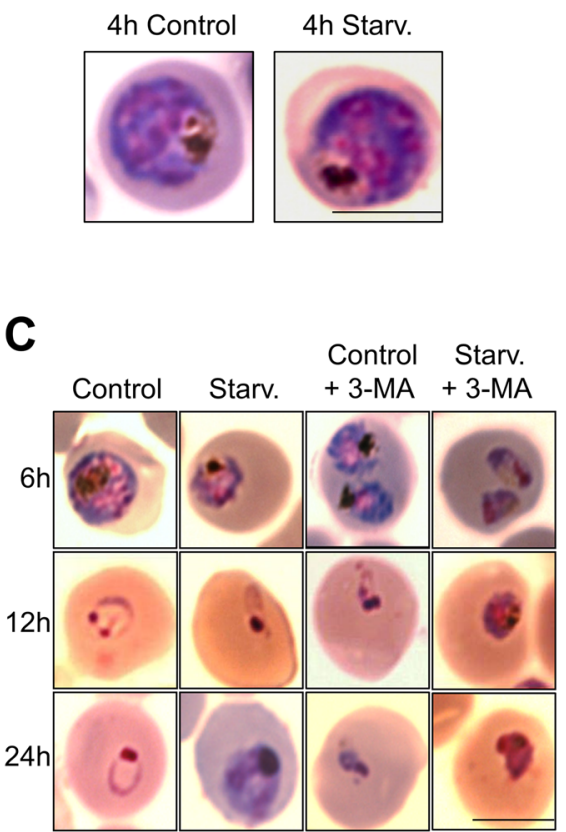

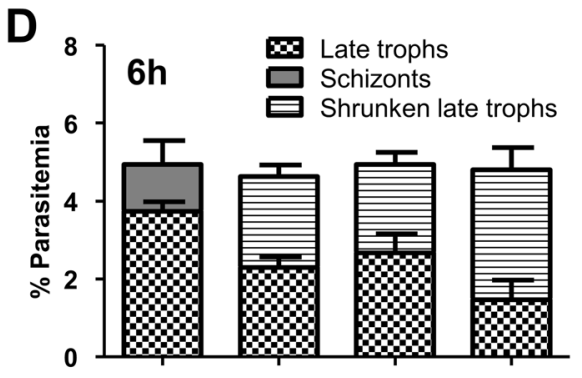

$\mathbf{E}$
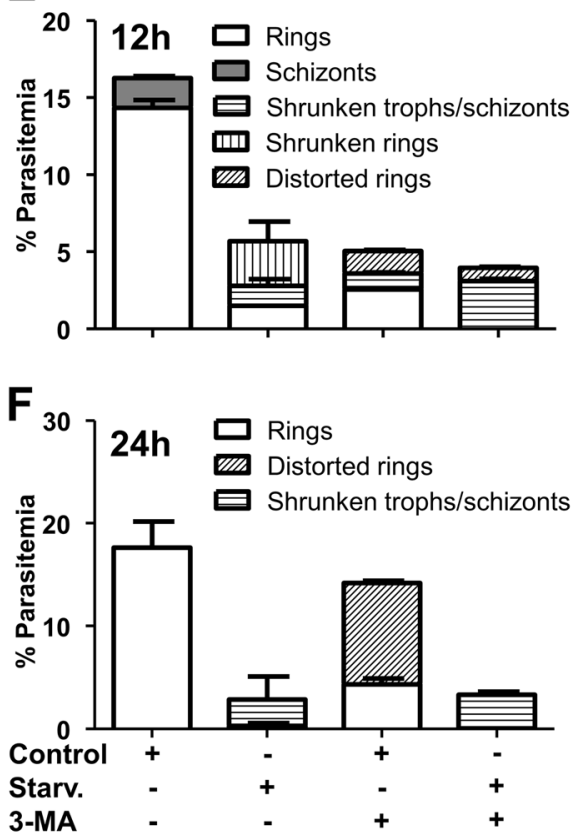

Fig. 3 Duration of starvation-induced autophagy determines parasite survival. a Immunoblot analysis of PfAtg8 expression levels upon 4 and 6 h starvation. Lysates from trophozoite stage parasites cultured in complete or starvation medium for 4 and $6 \mathrm{~h}$ were analyzed using immunoblotting by probing with custom generated anti-PfAtg8 antibodies. Uncropped blots are shown in Supplementary Figure 6. b Morphological features of control and $4 \mathrm{~h}$ starved parasites stained with Giemsa. Scale bar: $5 \mu \mathrm{m}$. c Prolonged starvation induces abnormal morphology. Parasites at late trophozoite stage were incubated in control, control + 3-MA, starvation or starvation + 3-MA media for 6, 12 and $24 \mathrm{~h}$. Parasite morphology was assessed in Giemsa stained smears. Scale bar:5 $\mu \mathrm{m}$. $\mathbf{d}$-f Graph representing parasites with starvation-induced morphological changes after 6, 12 and $24 \mathrm{~h}$ incubation in control, control + 3-MA, starvation or starvation + 3-MA media. Data represented is mean of 5 individual experiments. Number of parasites scored for morphology, $n=2500$. Error bars show standard deviation

contrast, there was a massive reduction in parasitemia in starved parasites (80\%), starved + 3-MA (82\%), and in unstarved parasites + 3-MA (30\%) (Fig. 3f).

Altogether, it is evident from these results that, upon long-term starvation, the parasites display abnormal morphology and stalled growth, supporting our notion that extended starvation results in down regulation of autophagy leading to cell death. As prolonged starvation and inhibition of autophagy resulted in an increase in the number of unhealthy parasites and a concomitant decrease in the appearance of healthy rings at the end of one developmental cycle, we wondered what the fate of these unhealthy parasites upon replenishment with fresh growth medium would be. For this purpose, treated or untreated parasites after 6 , 12 and $24 \mathrm{~h}$ of starvation were replenished with complete growth medium and the growth of these parasites were followed till the control parasites developed into subsequent stage. Progressive starvation and/or autophagy inhibition that resulted in stunted development and accumulation of unhealthy parasites were revived only to a certain extent compared to control, despite providing favorable nutrientrich conditions (Supplementary Figure S4).

\section{Long-term starvation inhibits reinvasion of host RBCs}

We next determined the effect of long-term starvation on invasion by following the development of late 

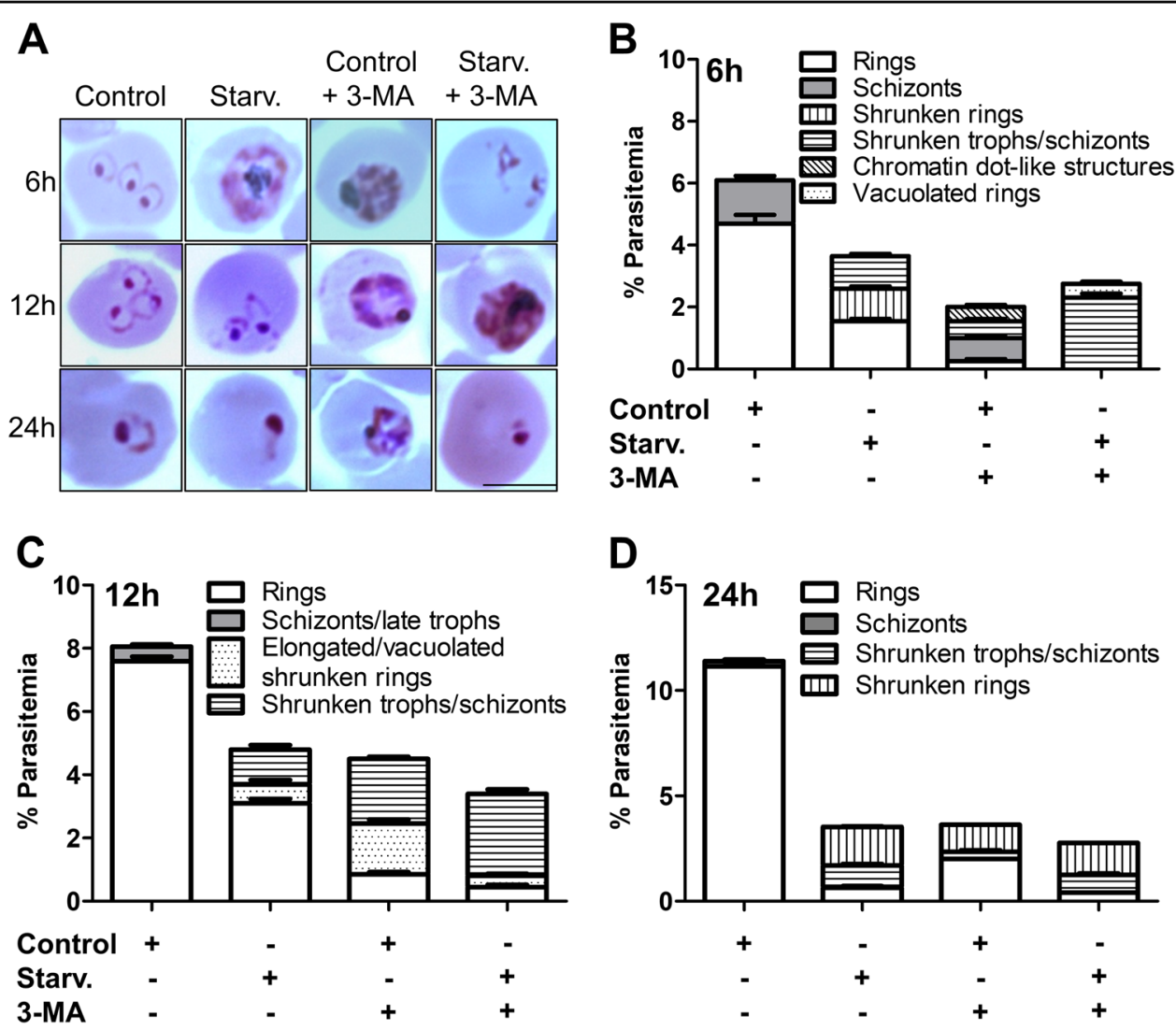

Fig. 4 Long-term starvation inhibits reinvasion of host RBCs. a Giemsa stained smears showing rings in representative iRBCs. Highly synchronized schizonts were incubated in complete or starvation medium in presence or absence of 3-MA for 6, 12 and $24 \mathrm{~h}$. Giemsa stained smears were assessed for number of rings and parasite morphology. Scale bar: $5 \mu \mathrm{m}$. $\mathbf{b}$ As described in (a), morphological analysis of the parasites after various treatments at different time points were carried out. Graphs representing \% parasitemia for these three time points are shown in $\mathbf{b}-\mathbf{d}$. The data represented are mean of 5 individual experiments. Number of parasites scored for rings, $n=2500$. Error bars show standard deviation

trophozoites/schizonts to rings in the next cycle. This progression was monitored by incubating late trophozoites/schizonts in complete (control) or starvation media, both in presence and absence of the autophagy inhibitor 3-MA for 6, 12 and $24 \mathrm{~h}$. While most of the control parasites developed into rings post $6 \mathrm{~h}$, many starved parasites remain arrested as late trophozoites/ schizonts (Control and Starv., 6 h, Fig. 4a). Quantitation of parasitemia after $6 \mathrm{~h}$ revealed a $40 \%$ decrease in starved parasites as compared to control (Control and Starv., $6 \mathrm{~h}$, Fig. 4b). Morphologically, while in control, $80 \%$ of the total parasites progressed to rings, only $40 \%$ healthy rings were observed with rest appearing as shrunken parasites under starved conditions (Control and Starv., 6 h, Fig. 4a, b). In parasites incubated in complete medium with 3-MA for $6 \mathrm{~h}$, there was a $63 \%$ decrease in parasitemia as compared to control, while presence of 3-MA in starvation medium reduced the parasitemia by $28 \%$ as compared to starvation medium alone (Control + 3-MA and Starv. + 3-MA, 6 h, Fig. 4b). Complete medium with 3-MA showed along with a few healthy rings, abnormal parasite morphology such as rings with multiple chromatin-like dots (17\%)and condensed schizonts with dark staining $(26 \%)$. In parasites incubated in starvation medium + 3-MA, none of the parasites appeared morphologically normal; $82 \%$ parasites were condensed schizonts and the rest were rings with chromatin-like dots in this group (Fig. 4a). The effect of 12 and $24 \mathrm{~h}$ starvation was much more deleterious on the invasion efficiency. As compared with control, there was a $37 \%$ drop in parasitemia at $12 \mathrm{~h}$ which after $24 \mathrm{~h}$ further decreased to $67 \%$ (Fig. 4c, d). Extended starvation also resulted in a temporal increase of unhealthy parasites. After $12 \mathrm{~h}, 95 \%$ of the parasites grown in complete medium were healthy rings which were only $63 \%$ in starved parasites (Fig. 4a). Shrunken trophozoites/schizonts and elongated/vacuolated rings constituted the rest of the starved parasites. Inhibition of autophagy by 3-MA during prolonged starvation (12 and $24 \mathrm{~h}$ ) further exacerbated the morphological abnormalities of the parasites resulting in reduced invasion fitness. Treatment with 3-MA in control medium caused a $42 \%$ decrease in parasitemia as compared to 


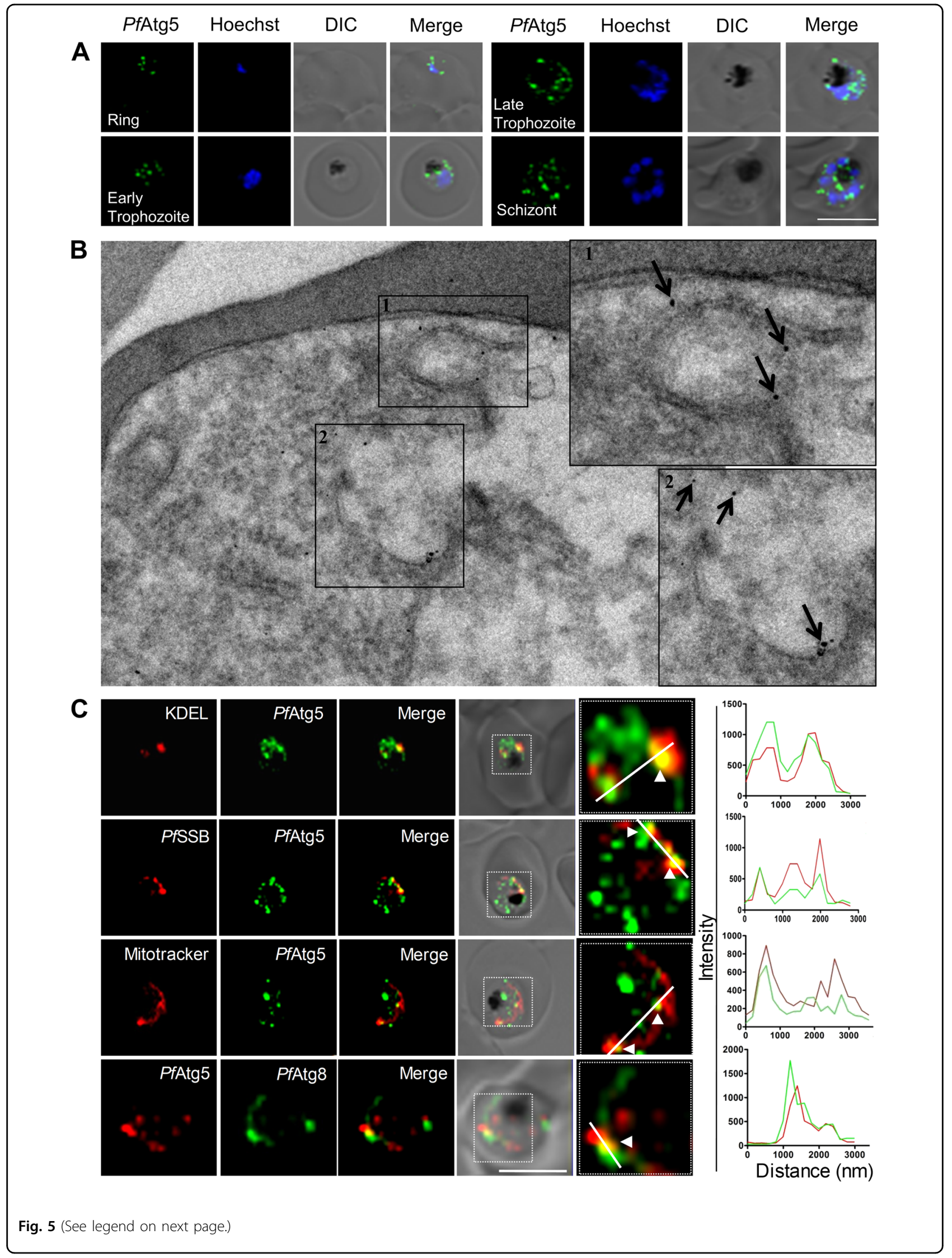


(see figure on previous page)

Fig. 5 The putative PfAtg5 is expressed throughout the intraerythrocytic cycle and appears as puncta in the parasite cytosol. a PfAtg5 immunofluorescence staining at various intraerythrocytic stages. Tightly synchronized parasites at ring, early trophozoite, late trophozoite and schizont stage were immunolabeled with anti-PfAtg5 antibodies. Representative image from at least 20 parasites is shown. Hoechst was used as a DNA marker. Scale bar: $5 \mu \mathrm{m}$. b RBCs infected with P. falciparum (8\% parasitemia) were analyzed by immunoelectron microscopy (immunogold and silver enhancement method) with antibody against PfAtg5. Insets 1 and 2 show enlarged images of Atg5 decorated double membrane structures. Scale bar: $0.2 \mu \mathrm{m}$. c Synchronized parasites at trophozoite stage were immunolabeled with anti-PfAtg 5 antibodies (1:400) along with various organelle markers: Anti-KDEL antibody (1:200) as an endoplasmic reticulum marker, Anti-PfSSB antibody (1:200) as an apicoplast marker, MitoTracker Red CMXROS (100 nM) as a marker for mitochondria and Anti-PfAtg8 antibody as a marker for autophagosomes. Hoechst was used as DNA marker; Scale bar: $5 \mu \mathrm{m}$

control at $12 \mathrm{~h}$, which further slid to $67 \%$ at $24 \mathrm{~h}$. Similarly, although there were barely unhealthy rings at 12 and $24 \mathrm{~h}$ in control, their numbers were $36 \%$ in 12 and $24 \mathrm{~h}$ in presence of 3-MA in complete medium. The unhealthy rings exhibited various deformities appearing as either shrunken, elongated with flattened nuclei, vacuolated or seen as chromatin-like dots without any ring structure. Remaining parasites in this group were late trophozoites with dense and condensed cytoplasm. There was a 58 and $74 \%$ drop in total parasitemia in parasites grown in starvation medium with 3-MA for 12 and $24 \mathrm{~h}$ respectively, as compared to control (Fig. 4c, d). Of the parasites that were cultured for 12 and $24 \mathrm{~h}$ in starvation medium in the presence of 3-MA, only $12-14 \%$ rings appeared normal. Rest of the abnormal parasites showed varied morphologies such as condensed schizonts with dark staining, or shrunken and vacuolated rings (Fig. 4a).

From these results, it is evident that prolonged starvation progressively results in reduced invasion. Blocking either basal or starvation-induced autophagy for an extended duration has a more telling effect on invasion as compared to starvation alone, further strengthening our notion that autophagy supports $P$. falciparum survival.

\section{Similar to PfAtg8, the putative PfAtg5 is expressed throughout the intraerythrocytic cycle as vesicles in the parasite cytosol}

As perturbation of autophagy showed dramatic effects on development, invasion and parasite survival, we wanted to explore further molecular details of autophagy in terms of autophagosome formation in P. falciparum. Atg8 has been extensively used as a late stage autophagosome marker in all model organisms and also in Plasmodium spp. However, early-stage autophagy markers such as Atg5 and Atg12 have not been explored in Plasmodium, although these have been identified in its genome ${ }^{5}$. Using antibodies against the putative PfAtg5, immunocytochemical staining revealed the presence of PfAtg5 decorated vesicles in the parasite cytosol in ring, trophozoite and schizont stages (Fig. 5a). Super resolution microscopy (3D-SIM) too revealed the presence of such structures (Supplementary Figure S1B). Interestingly, these PfAtg5 vesicles appeared similar to the PfAtg8 vesicles resembling autophagosomes (Figs. 1d, 2d). In addition, the presence of these Atg5 labeled double membrane vesicles was confirmed by immunoelectron microscopy (Fig. 5b). In complex eukaryotes, most autophagosomes appear to be generated from or are very close to, the endoplasmic reticulum. However, mitochondria, plasma membrane and Golgi have also been reported to contribute membranes for autophagosome generation ${ }^{13-15}$. We therefore investigated the colocalization between the early autophagosome marker PfAtg5 with various Plasmodium organelle markers such as KDEL (ER), SSB (apicoplast), MitoTracker Red (mitochondria) and also with the PfAtg8 (mature autophagosomes). We observed that PfAtg5 partially colocalized with ER, mitochondria, apicoplast and PfAtg8 (Fig. 5c).

\section{PfAtg5 expression too is induced by short-term starvation and inhibited by 3-MA}

Since, we observed that transient starvation-induced PfAtg8 expression while blocking autophagy reduced it, we wanted to see if $P f A t g 5$ expression pattern mimics that of PfAtg8. As seen for PfAtg8, we observed that PfAtg5 expression too increased upon starvation for brief periods (2 h, Fig. 6). This result was reflected in the appearance of PfAtg5 vesicles in late trophozoites/schizonts that were subjected to starvation in presence or absence of autophagy inhibition by 3-MA. Starvation caused an increase in abundance of PfAtg5 puncta while it diminished upon 3MA treatment in normal growth conditions or during starvation (Fig. 6).

\section{Discussion}

This study experimentally establishes the role of basal and starvation-induced autophagy in mediating $P$. falciparum development and invasion during its intraerythrocytic stages, demonstrating a prosurvival mechanism unlike reported for other apicomplexans such as Trypanosoma brucei ${ }^{16}$, and Toxoplasma gondii ${ }^{17,18}$. To address the role of autophagy in depth, we investigated the consequence of basal autophagy inhibition on blood stage parasite cycle over short-term and extended starvation. As 


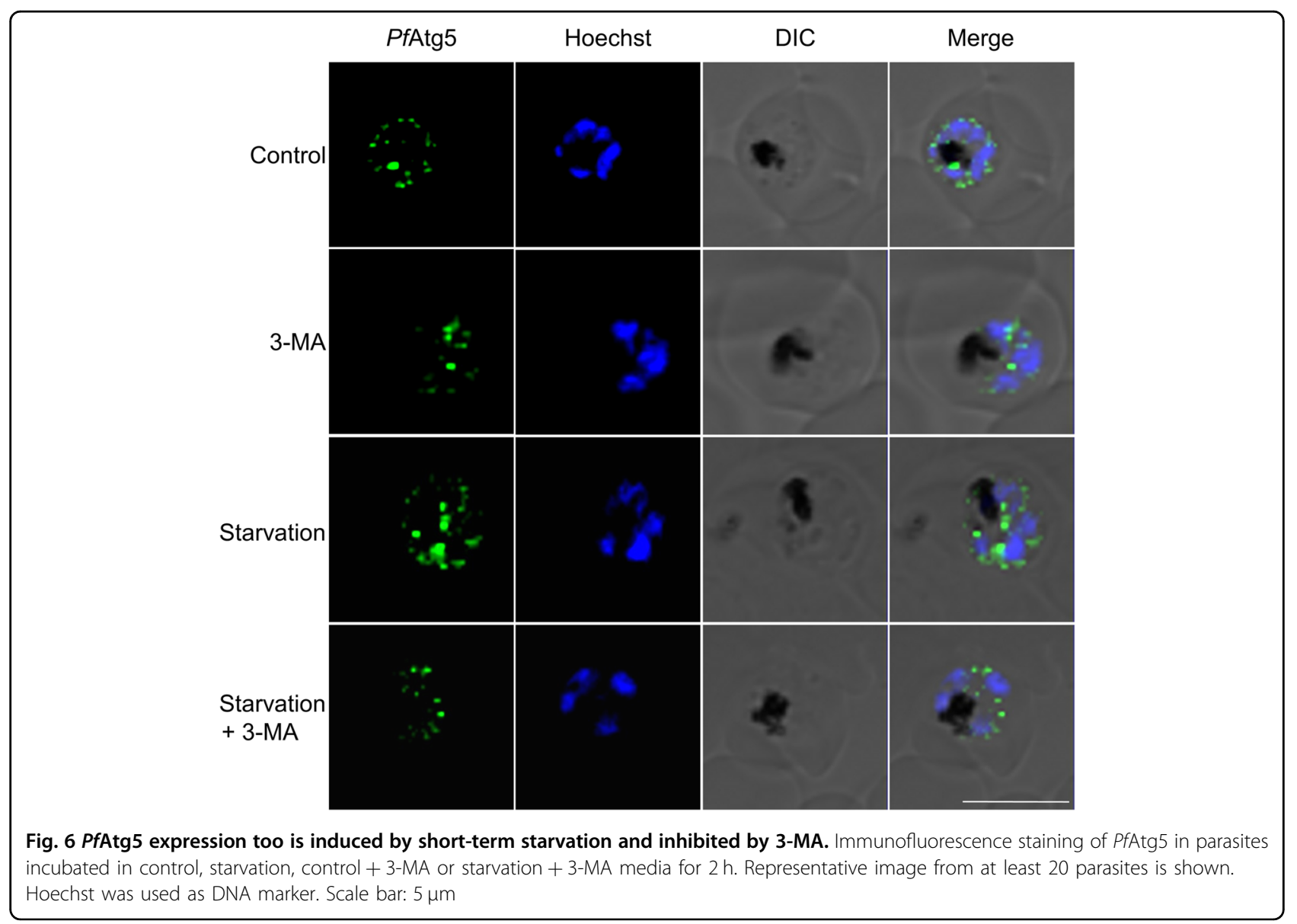

earlier studies using genetic manipulation approaches indicated that Plasmodium $A \operatorname{Atg} 8^{3}$ and PfAtg7 ${ }^{19}$ are essential for the parasite survival, we therefore chose to use alternative strategy such as pharmacological tools (autophagy inhibitors and inducers) to investigate the role of Plasmodium autophagy.

First, we addressed whether Plasmodium autophagy gets induced upon starvation as there have been conflicting reports. Studies on absence of autophagy induction are supported by the observation that bioinformatic analyses have not identified the complete set of the core autophagy machinery genes including Atg13, Atg9 and Atg16 11 , among others in P. falciparum, and in addition, starved parasites too have not shown induction in PfAtg8 levels ${ }^{4}$. However, a recent study showed increased colocalization of PfRab7and PfAtg8 positive vesicles upon $4 \mathrm{~h}$ of amino acid starvation ${ }^{8}$. We demonstrate that the parasite indeed responds to nutrient limitation by upregulating autophagy albeit for short duration and that this starvation-induced autophagy is context specific. While mild starvation induces autophagy enabling parasite development and invasion, prolonged starvation leads to decreased expression of PfAtg8, compromising autophagy which in turn leads to stalled growth and reduced invasion. Thus, our studies demonstrate induction of autophagy despite the lack of experimental evidence of a functional of TOR homolog in P. falciparum. Further, in the presumptive absence of Atg13, it would be interesting to explore that PfAtg1 participates in autophagy independently or in concert with FIP200 and Atg101 ${ }^{11}$.

Induction of autophagy is documented by monitoring the expression of the autophagosome marker PfAtg8 protein levels and the number and intensity of the puncta seen upon starvation. Again, in the literature the distribution of these PfAtg8 puncta is controversial. While some studies have shown them to localize exclusively to the apicoplast ${ }^{5}$, others have observed their presence within RBC cytosol ${ }^{6}$. We approached to resolve this issue by using heterologous (commercial LC3 A and B) as well as endogenous (PfAtg8) antibodies in immunoblotting and immunofluorescence techniques across all stages of the parasite erythrocytic cycle during starvation and/or autophagy inhibition (data with heterologous antibodies not shown). To further substantiate our results, we also employed another hitherto unexplored autophagosome marker in P. falciparum, the putative PfAtg5 and carried out similar analysis as mentioned for PfAtg8. In all these experiments, we consistently made two observations: one, 
transient or short starvation periods led to an upregulation of PfAtg8 and PfAtg5 expression with a concomitant increase in autophagosome numbers which was repressed upon autophagy inhibition; two, the distribution of the puncta resembling autophagosomes under all conditions and stages was restricted to the parasite cytosol alone. Discrepancies in non-induction of autophagy by starvation as well as redistribution of PfAtg8 vesicles reported in the past could be due to several reasons. Firstly, media used for such studies have varying compositions. While majority of the previous studies have used minimal medium $^{6}$ or Hanks' Balanced Salt Solution (HBSS) ${ }^{4}$, the starvation medium used by us is RPMI devoid of all amino acids and serum. Secondly, since maximum PfAtg8 expression was observed at late trophozoite stage, all our studies were carried out at this stage, while young/mid trophozoites were mostly used by others ${ }^{4,6}$. Also, our results show that autophagy induction is sustained only upto $4 \mathrm{~h}$ of starvation and beyond this period PfAtg8 levels are decreased leading to compromised parasite survival. In most of the studies carried out earlier, starvation was for $6 \mathrm{~h}$ (except in the studies of Tomlins et $\mathrm{al}^{8}$, where parasites were cultured in $1 \% \mathrm{O}_{2}, 3 \% \mathrm{CO}_{2}, 96 \% \mathrm{~N}_{2}$, and thus the results could be the combined effect of starvation and hypoxia).

Further, in absence of the full repertoire of core autophagy proteins as suggested by bioinformatics analyses, it has been difficult to envisage a functional autophagy pathway in this parasite. However, we find several lines of evidence in our studies that strongly point towards an operational and responsive autophagy pathway. First, we find that the parasite succumbs to starvation pressure when basal autophagy is inhibited. Second, brief starvation leads to transient increase in levels and puncta of both the autophagosomal markers, PfAtg8 as well as PfAtg5. Importantly, both PfAtg8 and PfAtg5 appear as puncta throughout the parasite cytosol, suggesting the presence of autophagosomes like vesicles that partially colocalizes with organelles such as mitochondria and ER that are known to supply membranes during autophagosome biogenesis ${ }^{15}$. Third, parasite cycle is sustained during short-term starvation and this cycle collapses upon autophagy inhibition. Fourth, prolonged starvation reduced the expression of the autophagy markers with concomitant parasite death.

Our characterization of the putative PfAtg5 unravels several unexpected and unique observations. As noted for mammalian $\operatorname{Atg} 5^{14}$, we show that PfAtg5 too appears as puncta in the cytoplasm of the parasite that partially colocalizes with PfAtg8, ER and mitochondria. Taken together, these results suggest that PfAtg 5 also participates in the autophagy pathway. Future work will be aimed at detailed studies in determining if a functional PfAtg5-PfAtg12 complex exists. At the molecular level, there are a few deviations from the classical Atg5-Atg12 seen in other species. The putative PfAtg5 is much longer protein as compared to its counterparts and although the lysine involved in conjugation with PfAtg12 is present at position 479 , in addition, a lysine residue is present at position $480^{5}$. Whether conjugation of PfAtg5 with the putative PfAtg12 in absence of the glycine at the $\mathrm{C}$ terminus of the PfAtg12 occurs or it conjugates with the inner glycine at 110 position of PfAtg12 needs further investigation. Interestingly, homolog of Atg16 has not yet been identified. Further work will shed light if this conjugation system and a functional canonical complex exists or whether PfAtg 5 tethers to the membrane vesicles independent of PfAtg12 as has been suggested for the yeast homolog ${ }^{20}$. Finally, in absence of a functional conjugation system, the possibility of these proteins engaging in other trafficking pathways cannot be ruled out.

Perhaps the more pertinent question to be asked is the need for autophagy in this parasite. The malaria parasite undergoes three major development stages during its intraerythrocytic cycle needing large and consistent supply of nutrients. The requirement of nutrients by the parasite during this asexual phase of life is reflected by the fact that almost $70-80 \%$ of hemoglobin is degraded by the parasite during the trophozoite stage to meet its demand for amino acids ${ }^{21}$. Also, in physiological context, during $P$. falciparum infection, the parasites get sequestered in blood vessels of various organs and as a result, the blood supply is obstructed and parasites do face severe nutrient limitation. Considering these scenarios and our results, it appears that autophagy serves as prosurvival mechanism under such conditions.

Altogether, our studies provide insights into the vital role of basal and starvation-induced autophagy in $P$. falciparum growth, development and invasion. For this, the parasite has evolved a unique autophagy machinery with a limited set of proteins with characteristic features. It is also tempting to suggest that these putative autophagy proteins may also have non-autophagy roles such as those ascribed for Atg8 in apicoplast biogenesis ${ }^{5}$. A comprehensive exploration to assign mechanistic roles of various proteins involved at different steps of autophagy in the parasite will need further exploration for developing novel therapeutics.

\section{Materials and Methods}

\section{$P$. falciparum parasite culture}

In vitro parasite culture procedures were approved by the Institutional Human Bioethics and Biosafety Review Committee of Jawaharlal Nehru Centre for Advanced Scientific Research, Bangalore, India. Plasmodium falciparum 3D7 strain was cultured in human $\mathrm{O}^{+}$erythrocytes at $37^{\circ} \mathrm{C}$ under $5 \% \mathrm{O}_{2}, 5 \% \mathrm{CO}_{2}$ and $90 \% \mathrm{~N}_{2}$ in RPMI 1640 (Sigma) supplemented with $27 \mathrm{mM}$ sodium bicarbonate, 
$11 \mathrm{mM}$ glucose, $0.37 \mathrm{mM}$ hypoxanthine, $10 \mu \mathrm{g} / \mathrm{ml}$ gentamicin and $10 \%$ heat inactivated human serum as described previously ${ }^{22}$. Parasite cultures were synchronized by sorbitol treatment ${ }^{23}$.

\section{Invasion assay}

Synchronized parasites at late schizont stage (38 h.p.i) were cultured in complete or starvation medium (RPMI 1640 without amino acids and serum, HyClone Laboratories Inc., South Logan, Utah) with or without $5 \mathrm{mM} 3-$ MA (M9281, Sigma). Invasion in the next cycle was checked by counting the number of rings in Giemsa stained thin smears using Axiostar plus microscope (Carl Zeiss).

\section{Immunoblot analysis}

For immunoblotting, synchronized parasites were separated from erythrocytes by treatment with $0.15 \%$ saponin in Phosphate Buffered Saline (PBS) for $15 \mathrm{~min}$ at $37^{\circ} \mathrm{C}$ followed by washes in cold PBS and centrifugation at 10,000 $\times$ $g$ for $10 \mathrm{~min}$. Saponin- treated parasites were suspended in SDS-PAGE sample buffer for $30 \mathrm{~min}$ at room temperature followed by sonication at 30 watts, 2 sec pulses three times at $5 \mathrm{~min}$ interval. Lysates were centrifuged at $16,000 \times g$ for $5 \mathrm{~min}$ and supernatant was collected. Protein was estimated by Bradford method ${ }^{24}$. Equal amount of protein $(30 \mu \mathrm{g})$ was resolved by $6 \mathrm{M}$ urea-SDS-PAGE gel for PfAtg8 and 10\% SDS-PAGE for PfAtg5 and blotted to PVDF membrane (Millipore). Primary antibodies used for immunodetection were custom generated rabbit anti-PfAtg8 (1:1,000) raised against a peptide IPVVCERANRSNLPHEKKK (corresponding to amino acids28-47) anti-PfAtg5 (1:1000) raised against a peptide corresponding to amino acids $170-190$ (Genemed synthesis, US) and anti- $\beta$-actin (1:3000, Sigma) diluted in 5\% skimmed milk/PBS. Both the custom antibodies were affinity purified. Their specificities were confirmed by peptide competition and by using uninfected RBC lysate. The commercial LC3 antibodies were LC3A (L8918, Sigma and 4599, Cell Signaling Technologies) and LC3B (3868, Cell Signaling Technologies). The signals were developed with an HRP-conjugated anti-rabbit secondary antibodies (1:3,000, Bangalore Genei) and Clarity Western ECL Substrate (Biorad) using Versa Doc (Biorad). Images were processed for brightness and contrast by Photoshop (Adobe Systems Inc.).

\section{Immunofluorescence Assay (IFA)}

Immunofluorescence assays were performed as described earlier ${ }^{25}$. Parasites were washed twice with PBS and fixed using 4\% paraformaldehyde (ProSciTech, Electron Microscopy-EM grade) and $0.0075 \%$ glutaraldehyde (EM grade) in PBS at room temperature for $30 \mathrm{~min}$. Fixed cells were washed 2 times with PBS and permeabilized using $0.1 \%$ Triton X-100 at room temperature for $3 \mathrm{~min}$. Cells were washed again twice with PBS followed by blocking with $3 \% \mathrm{BSA}$ at $4{ }^{\circ} \mathrm{C}$ for $1 \mathrm{~h}$. The primary antibodies were used at the following dilutions; rabbit anti-PfAtg8 (1:600), rabbit anti-PfAtg5 (1:400), mouse anti-SSB protein (1:400) and mouse anti-KDEL (1:400) in 3\% BSA in PBS for $1 \mathrm{~h}$ at room temperature. Secondary antibodies used were Alexa Fluor 488- and 568-conjugated goat anti-rabbit and goat anti-mouse (Molecular Probes) respectively at 1:200 dilutions in 3\% BSA for $1 \mathrm{~h}$ at room temperature. Nucleus was stained with Hoechst 33258 (Sigma). Parasites were mounted over the glass slide using ProLong Gold antifade mountant (Molecular Probes). Images were acquired with Carl Zeiss LSM700 or DeltaVision (Applied Precision, GE) microscope. Software used were Zen and SoftWoRx respectively using a $100 \mathrm{X}$ oil immersion phase contrast objective. DIC images were taken with polarized light.

\section{MitoTracker staining}

Parasites were incubated with $100 \mathrm{nM}$ MitoTracker Red CMXROS (M7512, Life Technologies) for $15 \mathrm{~min}$ at $37^{\circ} \mathrm{C}$, followed by washing the cells with PBS and fixing as described for IFA.

\section{PfAtg8-Atg5 colocalization studies by Zenon complex}

The parasites were fixed using $4 \%$ paraformaldehyde (ProSciTech, EM grade) and $0.0075 \%$ glutaraldehyde in PBS at room temperature for $30 \mathrm{~min}$. Fixed cells were washed twice with PBS and permeabilized with $0.1 \%$ Triton X-100 in PBS for $5 \mathrm{~min}$ at room temperature. Following PBS washes, cells were blocked in PBS containing 3\% BSA for $1 \mathrm{~h}$ at $4{ }^{\circ} \mathrm{C}$. This protocol was followed for the first primary antibody anti-PfAtg5 (1:200), Alexa Flour 568-conjugated goat anti-rabbit $(1: 200)$ was used as secondary antibody. Zenon rabbit IgG labeling was used to label PfAtg8. Zenon complex IgG 488 was prepared as recommended by the supplier (Zenon ${ }^{\circledR}$ Alexa Fluor ${ }^{\circledast} 488$ Rabbit IgG Labeling Kit, ThermoFisher Scientific) at a molar ratio of 6:1. Zenon complex thus formed was incubated for $1 \mathrm{~h}$ followed by fixation by $4 \%$ paraformaldehyde and $0.0075 \%$ glutaraldehyde in PBS at room temperature for $30 \mathrm{~min}$. Nuclear staining was performed with Hoechst 33258 (1:300) for $10 \mathrm{~min}$. Parasites were immediately mounted on the glass slide using VECTASHIELD (Vector Laboratories) mountant and imaging was carried out using Zeiss LSM 700 confocal microscope.

\section{Immunoelectron microscopy}

For immunodetection of PfAtg5 in infected RBCs, the previously described pre-embedding silver enhancement immunogold method ${ }^{26}$ was used with slight modifications.

The parasitized erythrocytes were fixed in $2 \%$ paraformaldehyde and $0.05 \%$ glutaraldehyde dissolved in $0.1 \mathrm{M}$ sodium phosphate buffer (PB) ( $\mathrm{pH}$ 7.4) for $2 \mathrm{~h}$ and then 
washed three times with PB. Then the cells were then resuspended in $2 \%$ agar and pelleted again. The cell pellets were immersed in $30 \%$ sucrose (wt/vol) overnight at 4 degrees. Immunolabeling was performed on 10-micron thick cryostat sections after blocking with $0.1 \%$ gelatin (wt/vol)-1\% BSA (wt/vol) in $0.02 \mathrm{M} \mathrm{PBS}$ for $30 \mathrm{~min}$ (blocking buffer 1) followed by another blocking in $1 \%$ NGS in PBS-gelatin-BSA buffer (blocking buffer 2).

Sections were incubated for $2 \mathrm{~h}$ at room temperature with 1:10 dilution of rabbit anti-PfAtg5 in blocking buffer 2 . After washing steps, sections were incubated with ultrasmall gold particles (1:50 dilution, Electron Microscopy Sciences) for $4 \mathrm{~h}$ at room temperature, followed by washing and post fixation with $2 \%$ glutaraldehyde for $20 \mathrm{~min}$. Silver enhancement (R-GENT SE-EM, Electron Microscopy Sciences) was performed en-bloc for 10 minutes followed by dehydration in graded series of ethanol. Finally, the sections were embedded in Epon 812 resin and allowed to polymerize overnight at $60^{\circ} \mathrm{C}$. Ultrathin sections $(70 \mathrm{~nm}$ thick) were cut on RMC ultramicrotome, stained with $1 \%$ Uranyl acetate and imaged in Tecnai G2 20 twin (FEI) transmission electron microscope.

\section{Acknowledgements}

N.S. and R.M. wish to thank JNCASR for intramural support and Prof. M.R.S. Rao for constant encouragement. R.M. is supported by Wellcome Trust/ Department of Biotechnology (DBT), India Alliance Intermediate Fellowship (500159-Z-09-Z) and N.S. acknowledges Indian Council of Medical Research (India) grant. This work was also supported by a joint grant from DBT to N.S. and R.M. We thank Dr. Suman Dhar (JNU, Delhi) for the kind gift of anti-SSBP antibodies, Prof. K. Suguna and Nikhil (IISC, Bangalore) for modeling the docking of PfAtg1 and MRT68921 and Prof. A. Surolia (IISC, Bangalore) for helpful discussions and critical reading of the manuscript.

\section{Author details}

${ }^{1}$ Molecular Biology and Genetics Unit, Jawaharlal Nehru Centre for Advanced Scientific Research, Jakkur, Bangalore 560064, India. ${ }^{2}$ CSIR-Institue of Genomics and Integrative Biology, Room 223, Sukhdev Vihar, Mathura Road, New Delhi 110025, India. ${ }^{3}$ Electron Microscopy Laboratory, Department of Neuropathology, National Institute of Mental Health and Neuro Sciences, Bangalore 560029 Karnataka, India

\section{Conflict of interest}

The authors declare no potential conflict of interest.

\section{Publisher's note}

Springer Nature remains neutral with regard to jurisdictional claims in published maps and institutional affiliations.

The online version of this article (https://doi.org/10.1038/s41420-018-0107-9) contains supplementary material, which is available to authorized users.

Received: 14 June 2018 Revised: 29 August 2018 Accepted: 5 September 2018

Published online: 03 October 2018

\section{References}

1. Klionsky, D. J. et al. A comprehensive glossary of autophagy-related molecules and processes (2nd edition). Autophagy 7, 1273-1294 (2014).

2. He, C. \& Klionsky, D. J. Regulation mechanisms and signaling pathways of autophagy. Annu. Rev. Genet. 43, 67-93 (2009).

3. Brennand, A. et al. Autophagy in parasitic protists: Unique features and drug targets. Mol. Biochem. Parasitol. 177, 83-99 (2011).

4. Navale, R., Atul, Allanki, A. D. \& Sijwali, P. S. Characterization of the autophagy marker protein Atg8 reveals atypical features of autophagy in Plasmodium falciparum. PLOS ONE 9, e113220 (2014).

5. Kitamura, K. et al. Autophagy-related Atg8 localizes to the apicoplast of the human malaria parasite Plasmodium falciparum. PLOS ONE 7, e42977 (2012).

6. Cervantes, S. et al. The multifunctional autophagy pathway in the human malaria parasite Plasmodium falciparumAutophagy 10, 80-92 (2013).

7. Sinai, A. P. \& Roepe, P. D. Autophagy in Apicomplexa: a life sustaining death mechanism? Trends Parasitol. 28, 358-364 (2012).

8. Tomlins, A. M. et al. Plasmodium falciparum ATG8 implicated in both autophagy and apicoplast formation. Autophagy 9, 1540-1552 (2014).

9. Jayabalasingham, B., Bano, N. \& Coppens, I. Metamorphosis of the malaria parasite in the liver is associated with organelle clearance. Cell Res. 20, 1043-1059 (2010)

10. Petherick, K. J. et al. Pharmacological Inhibition of ULK1 kinase blocks mammalian target of rapamycin (mTOR)-dependent autophagy. J. Biol. Chem. 290, 11376-11383 (2015)

11. Hain, A. U. P. \& Bosch, J. Autophagy in Plasmodium, a multifunctional pathway? Comput. Struct. Biotechnol. J. 8, e201308002 (2013).

12. Klionsky, D. J. et al. Guidelines for the use and interpretation of assays for monitoring autophagy. Autophagy 8, 445-544 (2012).

13. Suzuki, K. et al. The pre-autophagosomal structure organized by concerted functions of APG genes is essential for autophagosome formation. EMBO J. 20, 5971-5981 (2001)

14. Mizushima, N. Dissection of autophagosome formation using Apg5-deficient mouse embryonic stem cells. J. Cell Biol. 152, 657-668 (2001).

15. Shibutani, S. T. \& Yoshimori, T. A current perspective of autophagosome biogenesis. Cell Res. 24, 58-68 (2013).

16. Li, F. J. et al. A role of autophagy in Trypanosoma brucei cell death. Cell. Microbiol. 14, 1242-1256 (2012).

17. Ghosh, D., Walton, J. L., Roepe, P. D. \& Sinai, A. P. Autophagy is a cell death mechanism in Toxoplasma gondii. Cell. Microbiol. 14, 589-607 (2012).

18. Besteiro, S. Which roles for autophagy in Toxoplasma gondii and related apicomplexan parasites? Mol. Biochem. Parasitol. 184, 1-8 (2012).

19. Walker, D. M. et al. Plasmodium falciparum erythrocytic stage parasites require the putative autophagy protein PfAtg7 for normal growth. PLOS ONE 8, e67047 (2013).

20. Romanov, J. et al. Mechanism and functions of membrane binding by the Atg5-Atg12/Atg16 complex during autophagosome formation. EMBO J. 31 4304-4317 (2012)

21. Rosenthal, P. J. Molecular Approaches to Malaria Ch. 16 (ASM Press, Washington, DC, 2005).

22. Trager, W. \& Jensen, J. B. Human malaria parasites in continuous culture. Science 193, 673-675 (1976).

23. Lambros, C. \& Vanderberg, J. P. Synchronization of Plasmodium falciparum erythrocytic stages in culture. J. Parasitol. 65, 418-420 (1979).

24. Bradford, M. M. A rapid and sensitive method for the quantitation of microgram quantities of protein utilizing the principle of protein-dye binding. Anal. Biochem. 72, 248-254 (1976).

25. Tonkin, C. J. et al. Localization of organellar proteins in Plasmodium falciparum using a novel set of transfection vectors and a new immunofluorescence fixation method. Mol. Biochem. Parasitol. 138, 251-253 (2004).

26. Melo, R. C., Morgan, E., Monahan-Earley, R., Dvorak, A. M. \& Weller, P. F. Preembedding immunogold labeling to optimize protein localization at subcellular compartments and membrane microdomains of leukocytes. Nat. Protoc. 9, 2382-2394 (2014). 